\title{
Anti-respiratory passage virus of Chinese herbal medicine
}

\author{
Zhiqiang Wang, Yi Zhu* \\ Department of Acupuncture and Massage, Nanjing University of Traditional Chinese Medicine, Nanjing, China; \\ wangzhiqiangshow5@163.com; *Corresponding Author: zhuyi1010@,163.com
}

Received 20 April 2010; revised 20 October 2010; accepted 22 October 2010

\begin{abstract}
Influenza virus and respiratory syncytial virus infection of viral respiratory disease has become a major killer of mankind, but there is no effect of vaccines and therapeuticmdrugs. Chinese herbs can inhibit the proliferation and strengthen the human immune virus, and so on to play a very good antiviral activity. Singleflavor compound in accordance with two different drugs and the object of study, the herbal anti-influenza virus and respiratory syncytial virus, a comprehensive overview of research and clinical outlook of the Chinese herbs to good use.
\end{abstract}

Keywords: Antiviral; Chinese Herbal Medicine; Influenza Virus; Respiratory Syncytial Virus

\section{INTRODUCTION}

Viral respiratory disease is a class of common disease that have high incidence, all ages are susceptible, infection in children and the elderly often lead to more serious complications, a pandemic would bring a high mortality rate, that to be a serious harm to human health. So far western medicine has not found a way that have a good efficiency and have no toxicity. But anti-viral drugs of chinese herbal medicine use natural medicine, which has many features that chemical drugs do not have, and have extensive source, low price, easy to spread. Because of the above reasons, the present stage of Chinese medicine is committed to anti-virus research, to explore this vast treasure house of Chinese medicine, which has obviously found a lot of antiviral drugs. This passage review on Chinese herbal medicine research about the influenza viruses and respiratory syncytial virus in the last ten years.

\section{INFLUENZA VIRUS}

Influenza viruses easily lead to acute respiratory dis- ease, not only affect people's normal life, but also easily lead to serious complications. Although the inactivated vaccine and live attenuated vaccines have achieved some results, but we still did not overcome the difficulties of the surface glycoprotein of influenza virus variation to the traditional vaccine [1]. And because the current drugs used to combat influenza based mainly synthetic drugs such as amantadine, rimantadine and zanamivir, But it has problem of toxic side effects and inconvenient to take the issue $[2,3]$. Therefore, it is necessary to develop low toxicity and effective Chinese herbal medicine to Anti-influenza virus.

\subsection{Single Medicine Research}

Zheng Qun [4] et al used mouse lung virus with influenza virus infection in mice adapted strain A/FM/1/47 pneumonia model to study catechu extract on influenza virus infection in the body on body immune function, but also on Delayed-type hypersensitivity (DTH) and the impact of neutralizing antibodies. The results showed catechu extract $12.5 \mathrm{~g} / \mathrm{L}$ mice infected with pneumonia, which has a good protective effect of the death, catechu extract $6.25 \mathrm{~g} / \mathrm{L}$ can significantly prolong the average survival time of infected mice. This indicates that the catechu extract in mice with effective anti-influenza virus effect. At the same time,research also shows that catechu extract can enhance the influenza virus infection and cellular immunity and humoral immunity.

Guo Yanhua [5] et al used mouse lung virus with influenza virus infection in mice adapted strain A/FM/1/47 pneumonia model to research Notopterygium influence on influenza virus pneumonia in mice and protective effect of the death of mice preventive effect of influenza virus. The research found Notopterygium extract high, medium dose group can effectively reduce the mortality rate of mice infected with the virus, the high dose can directly kill influenza virus in mice lungs and lower hemagglutination titer, and the protective effect to death of mice infected with the virus is better than ribavirin, role in the control group with the same effect of Shuang- 
huanglian $(\mathrm{P}>0.05)$.

Chen Zhiwei [6] used microscopic to determine the differences in Morphology of different treatment Radix to red blood cell infection of influenza virus. Discussing the mechanism of aqueous extract of Radix inhibition Cell binding activity of influenza A virus by capillary electrophoresis combined with microscopic examination and hemagglutination titer index. Experiments show that water extract of Radix inhibited virus binding to red blood cells in solution, is a board dominated by blue fine roots of red cell's own protective effect, which effectively inhibit the influenza virus binding to cells to achieve the anti-virus purposes.

The cell culture technique to ribavirin as a positive control to observe the anthraquinone compounds on influenza virus cytopathic (CPE) in vitro. Found that half of its anti-influenza virus inhibitory concentration (IC50) was $122.4 \mu \mathrm{g} / \mathrm{ml}$, half of the toxic concentration (TC50) to $226.1 \mu \mathrm{g} / \mathrm{ml}$, therapeutic index (TI) was 1.9 , and the inhibition of influenza virus, the amount of obvious effective response relationship, and the large amount of inhibition of the virus on a large [7].

Litchi flavonoids have a good appreciation of the influenza virus inhibited the toxicity of Litchi flavonoids lower than ribavirin, and in $160 \mu \mathrm{g} / \mathrm{ml}$ or more, to the extent of influenza virus cytopathic effect inhibition up to $50 \%$ or more, that is, at a certain concentration is Litchi flavonoids relatively safe and effective antiviral drugs [8].

\subsection{Compound Medicine Research}

Wang Baohua [9] et al infected mice in each group by the dilution of influenza virus FM1 intranasally, and establish infection model of influenza virus to do research on Ag Alice Cai Gui Decoction on the role of influenza virus replication with real-time and accuracy of real-time fluorescence quantitative polymerase chain reaction (PCR). They establish a normal control group, a virus model group, a ribavirin group and a Tang Yin Qiao Cai Gui group. It was found that the virus model was amplified with the curve of $\mathrm{Ct}$ model group were $(19.49 \pm 1.40),(21.02 \pm 4.25),(15.80 \pm 4.18)$ respectively, and the test proves positive for influenza virus pathogens. Compared with the model group with the virus replication capacity $(128973.40 \pm 18.14)$, the amount of viral replication of ribavirin group (33327.29 $\pm 19.56)$ decreased with significantly difference $(\mathrm{P}<0$. 05 ), and the Tang Yin Qiao Cai Gui-group virus replication volume $(79104.28 \pm 2.52)$ decreased also with significant difference $(\mathrm{P}<0.05)$. The above data shows that Yinqiao Cai Gui Decoction has obvious inhibition on pulmonary influenza virus of mice infected by proliferation of influenza virus.
Mei-Juan Chen [10] et al mice with lung adapted strain of influenza virus (FM1) nasal instillation of infected BALB/C mice replication animal model to record the survival time of mice in order to measure the lung index. They also use FM1 infected MDCK cell lines (MDCK) as the experimental system to observe the degree of cytopathic effect, and thus to understand the effect of Yin huang Injection against FM1. The vitro experiments showed that after treatment before the virus infected, the amount of Yin huang Injection about 0.11 $\mathrm{mg} / \mathrm{ml}$ can inhibit FM1 MDCK cells on pathological damage, while before the treatment but after the virus infected, the amount of about $0.22 \mathrm{mg} / \mathrm{ml}$ can inhibit FM1 more on MDCK cells injury. The animal experiments showed that only a small doses of intramuscular injection of Yin huang, can only decrease the pulmonary index values $(\mathrm{P}<0.05)$, but can significantly reduced lung index $(\mathrm{P}<0.01)$ with medium and large doses and extend the survival time $(\mathrm{P}<0.05)$. At the same time, the intravenous infusion of small doses will only reduce pulmonary index values $(\mathrm{P}<0.05)$, while the medium and large doses can significantly reduce lung index and prolong survival time $(\mathrm{P}<0.01)$.

\section{RESPIRATORY SYNCYTAIL VIRUS}

Respiratory syncytial virus is one of the important pathogens that can cause Infant lower respiratory tract infection. For a long time, there is no effective medicine against RSV infection, and even if there are formalin inactivated vaccine, live attenuated vaccine technology and the development of subunit vaccine, Genetically engineered vaccine, DNA vaccine, but not safe or ineffective [11-12]. But many herbs by acting on different targets and to achieve better antiviral.

\subsection{Single Medicine Research}

Patrinia effective part of anti-virus isolated AP3 by Chinese medicine decoction, ethanol extraction and macroporous resin chromatography. AP3 on the inhibition of RSV obvious dose-effect relationship in different concentrations, The longer the role of , the more the inhibitory effects. Its effect primarily through inhibition of RSV respiratory syncytial virus into the cell membrane, inhibite virus replication in cells [13].

Liu Zhao [14] observe virus induced cytopathic effect and MTT assay of drug activity against RSV, calculate the inhibition of drugs on disease rates and half inhibitory concentration, antiviral drugs adsorbed, drugs can kill the virus directly and inhibition of the virus in cell biosynthesis, exploration activity in isatis monomer mechanism of anti-RSV in three aspects. The results showed no direct isatidis monomer inactivation of RSV, 
can not prevent the adsorption of RSV, Which can inhibit RSV in Hep-2 cells the biosynthesis. So it shows isatidis monomer safely and efficiently inhibit RSV in Hep-2 cell proliferation, inhibition occurred after viruses invade cells.

Chrysanthemum aqueous extract have good inhibitory effect of RSV In vitro. It can play a role in many areas, it not only inactivates viruses directly in incubated with virus, but also inhibites virus adsorption and penetrate the cell membrane infected cells, meanwhile it can remove the virus which have penetrated cells [15].

\subsection{Compound Medicine Research}

$\mathrm{Xu} \mathrm{Xia}$ [16] et al used JinbeiqingfeiKeLi containing honeysuckle, forsythia, Eustoma, loquat, gypsum and other 10 Chinese herbs to act on the respiratory syncytial virus pneumonia in animal models of indirect elections in the $0.18-2.89 \mathrm{~g} / \mathrm{kg} 5$ doses of particles observed Jinbeiqingfei infection in mice, respiratory syncytial virus pneumonia of indicated doses of the drug began to work in $0.36 \mathrm{~g} / \mathrm{kg}$ and was compeletly inhibited up to 1.45 $\mathrm{g} / \mathrm{kg}$ doses in lung tissue proliferation, which can save animals from infection with respiratory syncytial virus pneumonia, the effect was superior than ribavirin dose from in $0.72 \mathrm{~g} / \mathrm{kg}$ doese of JinbeiqingfeiKeLi.

Liao Hui [17] et al took the human embryonic lung fibroblast into groups, then respiratory syncytial virus attacked those groups, after that the electron microscopies in each group recorded the changes of ultrastructure of human embryonic lung fibroblasts. The results showed that the viral infection increases the nucleus, nuclear cytoplasmic ratio increase, nucleolar condensation, nuclear membrane sag into the interlayer, the formation of inclusion bodies, mitochondrial condensation, expansion of rough endoplasmic reticulum, lysosomes increased, chromatin condensation, cellvirus particles can be seen outside. After infection with serum treatment group, the nuclei enlarged, nucleolus condensation, expansion of rough endoplasmic reticulum, mitochondria condensation, but to a lesser extent. Confirmed that when human embryonic lung fibroblast cells infected by RSV, the cell ultrastructural changes may occur, Qingfei oral liquid contained in serum can reduce those changes and the effect is move obvious when it is ussed at the moment of infection than after that.

\section{CONCLUSIONS}

Sum up all these things above, Chinese herbal medicine have a good inhibition on the respiratory virus. It is reflected in the inhibition of many aspects such as virus inhibition value and improve the immune function, and compared with western medicine it has low toxicity, those are the advantages of herbal anti-virus. Chinese herbs can act on different targets, in recent years the study of Chinese herbal medicine shifted from single medicine to compound medicine, The introduction of a large number of high technology to study the antiviral mechanism of Chinese herbal medicine. But the study also is not deep enough, the antiviral mechanism of Chinese herbal medicine is not into the system, and there are many problems in the study of chinese compound medicine, such as chinese herbal medicine has many different components, and it will be interaction between different components, this brings us to the question that the research results will be different in a different environment, different concentrations or using different extraction techniques. So I think the Chinese herbal medicine research must be combined with the latest technology, Learn from the experience of Western anti-virus, do dore detailed studies of chinese medicine, able to extract the active ingredients, truly "learn the essence, discard the dregs", to make of the new formulation for clinical.

\section{REFERENCES}

[1] Li, R.Q. and Li Y. (2004) The research progress of influenza vaccination. China Journal of Experimental and Clinical Virology, 6, 198-201.

[2] Hu, W.F., Zhang, Y.P. and Qiu, D. (2006) The research about the effect of compound large fruit soft capsules sericea anti-influenza virus in vitro. Lishizhen Medicine and Materia Medica Research, 10, 167.

[3] Ye, M. (2000) Anti-flu drugs-zanamivir. Chinese Pharmaceutical Journal, 3, 136.

[4] Zheng, Q., Ping G.L. and Zhao, W.M. (2004) Catechu extract on influenza virus infection in immune function in mice. Capital University of Medical Journal, 25, 180182.

[5] Guo, Y.H., Sha, M., Meng, X.S., et al. (2005) Anti-virus research traditional Chinese medicine Notopterygium. State Medical Sinopharm Shizhen, 16, 198-199.

[6] Chen, Z.W., Wu, L.W., Liu, S.T., et al. (2006) Capillary electrophoresis banlangen water extract mechanism of action of anti-influenza virus. China Journal of Traditional Chinese Medicine, 31, 1715-1719.

[7] Liang, R.G., Luo, W.S., Li, L.Y., et al. (2006) Rhubarb anthraquinones in vitro anti-influenza virus in vitro. China Medical, 19, 96-398.

[8] Luo, W.S., Gong, S.J., Liang, R.G., et al. (2006) Litchi flavonoids in vitro anti-influenza virus in vitro. China Journal of Traditional Chinese Medicine, 31, 1379-1380.

[9] Wang, B.H., Li, S.M., Zuo, J.L. (2009) Tang Yin Qiao Chaigui effect of anti-influenza: A virus experimental study. Journal of Beijing University of Chinese Medicine, 32, 540-543.

[10] Chen, M.J., Ge, L., Xiao, S.H., et al. (2007) Silver yellow liquid inside and outside the injection of anti- influenza virus in experimental study. State Medical Sinopharm Shizhen, 18, 591-592. 
[11] Li, H. (2007) The treatment respiratory progress of syncytial virus pneumonia. Jiangxi Traditional Chinese Medicine, 38, 76-77.

[12] Su, R. and Wang, L.B. (2006) Respiratory syncytial virus vaccine study. International Journal of Pediatrics, 33, 187-189.

[13] Li, S.S., Li, H.Y., Pu, Y.G., et al. (2004) The study of patrina an effective part of anti-viral grass respiratory syncytial virus in vitro inhibitory effect. Chinese Journal of Epidemiology, 25, 150-153.

[14] Liu, Z., Yang, Z.Q. and Xiao, H. (2009) Daqingye effective monomer anti-respiratory syncytial virus in rats.
State Medical Sinopharm Shizhen, 20, 1977-1979.

[15] Zhang, Z.Y., Fang, X.P., Diao, Z.H., et al. (2006) The study of Chrysanthemum inhibition of respiratory syncytial virus in vitro. PLA Pharmaceutical Journal, 22, 273-276.

[16] Xu, X., Sui, Z.Y. and Sui, L.Y. (2007) Kanagai Qingfei particles in vivo anti-respiratory syncytial virus in rats. State Medical Sinopharm Shizhen, 18, 403-404.

[17] Liao, H., Wang, L. and Wang, S.C. (2008) Respiratory syncytial virus infection in cell ultrastructure changes and Qingfei oral liquid drug-containing serum of its role. Liaoning Journal of Traditional Chinese Medicine, 35, 656-657. 